\title{
Contrast Detection Learning Improves Visual Contrast Sensitivity of Cat
}

\author{
HUA Tian-Miao*, WANG Zhen-Hua, XU Jin-Wang, DIAO Jian-Gang \\ (School of Life Sciences, Anhui Normal University, Wuhu 241000, China)
}

\begin{abstract}
Psychological studies on human subjects show that contrast detection learning promote learner's sensitivity to visual stimulus contrast. The underlying neural mechanisms remain unknown. In this study, three cats (Felis catus) were trained to perform monocularly a contrast detection task by two-alternative forced choice method. The perceptual ability of each cat improved remarkably with learning as indicated by a significantly increased contrast sensitivity to visual stimuli. The learning effect displayed an evident specificity to the eye employed for learning but could partially transfer to the naïve eye, prompting the possibility that contrast detection learning might cause neural plasticity before and after the information from both eyes are merged in the visual pathway. Further, the contrast sensitivity improvement was evident basically around the spatial frequency (SF) used for learning, which suggested that contrast detection learning effect showed, to some extent, a SF specificity. This study indicates that cat exhibits a property of contrast detection learning similar to human subjects and can be used as an animal model for subsequent investigations on the neural correlates that mediate learning-induced contrast sensitivity improvement in humans.
\end{abstract}

Key words: Visual; Contrast detection; Learning; Contrast sensitivity; Cat

\section{对比度检测学习提高猫的视觉对比敏感度}

\author{
华田苗*，王振华，徐金旺， 习建刚 \\ (安徽师范大学 生命科学学院, 安徽 芜湖 241000)
}

摘要: 对人的心理学研究结果显示, 对比度检测学习可提高学习者对视觉刺激的对比敏感度, 但其潜在的神经机制尚不清 楚。该研究用二选一 (two-alternative forced choice) 方法训练 3 只猫 (Felis catus) 通过单眼进行对比度检测学习, 发现每只猫 对视觉刺激的对比敏感度随着训练而显著提高。该学习效果虽然对训练眼有明显的特异性, 但部分学习效果可以传递给非训练 眼, 提示对比度检测学习可能会引起双眼信息汇聚前后的视觉中枢的神经可塑性。另外, 猫视觉对比敏感度的提高主要发生在 训练刺激的空间频率附近, 表明对比度检测学习具有一定的空间频率选择性。该研究结果显示, 猫对视觉刺激的对比度检测学 习表现出与人类相似的特性, 因此可以作为模式动物来研究人类学习诱导的视觉对比敏感度升高的神经机制。

关键词: 视觉; 对比度检测; 学习; 对比敏感度; 猫

中图分类号: Q42;Q954.67+1;Q959.838.05 文献标志码: A 文章编号: 0254-5853-(2010)02-0155-08

Human perceptual learning, including orientation discrimination, motion perception, contrast detection and the spatial frequency (SF) discrimination has been well documented (Gray \& Regan, 1998; Grove \& Regan, 2002; Karni \& Sagi, 1991; Lages \& Treisman, 1998; Matthews et al, 1999; Matthews et al, 2001; Niebauer \& Christman, 1999; Rivest et al, 1997; Sally \& Gurnsey, 2003; Sowden et al, 2002; Vimal, 2002). The long-lasting improvement through learning was highly specific to stimulus parameters, retinal locations and/or even the eye employed during learning (Gilbert, 1994; Karni \& Sagi, 1991; Sowden et al, 2002). Such specificity of learning was generally interpreted as an evidence of learning-induced plasticity at early stages of visual information processing, such as the primary visual cortex (Dosher \& Lu, 1999; Gilbert, 1994; Karni \& Sagi,

Received date: 2009-09-11; Accepted date: 2010-02-01

Foundation items: Supported by Natural Science Foundation of Anhui Province (070413138); the foundation of Key Laboratory of Anhui Province and the Key Research Foundation from Education Department of Anhui Province (KJ2009A167)

收稿日期：2009-09-11；接受日期：2010-02-01

*通讯作者 (Corresponding author), E-mail: tianmiaohua@gmail.com 
1991). However, the neural mechanism underlying such perceptual learning is poorly understood though a progress has been achieved in psychological paradigms (Dosher \& Lu, 1998; Dosher \& Lu, 1999). A main difficulty that faced this issue may lie in the limitation of applying powerful research approaches, such as electrophysiological recording and brain lesions, on human subjects.

A few of studies have been done in adult monkeys, attempting to locate the brain region responsible for the psychological performance improvement in stimulus orientation discrimination. It was believed that orientation discrimination learning could modify the orientation tuning property of neurons at least in some areas of the visual cortex. However, results from different research groups were contradictory (Ghose et al, 2002; Raiguel et al, 2006; Schoups et al, 2001; Yang \& Maunsell, 2004).

Whether and how the learning of visual tasks other than orientation discrimination, such as contrast detection, affects the tuning property of visual cortical cells has not been explored so far. All visual targets in the real world are perceived in varied contrast. It's now widely accepted that contrast sensitivity, which reflects how well we see, is one of the best indices for visual quality evaluation (Altangerel et al, 2006; Ginsburg, 2006; Piermarocchi et al, 2006; Riusala et al, 2003). Experiments on human subjects showed that practising contrast detection could significantly improve contrast sensitivity of visual perception. The learning effect was proven to be specific for stimulus $\mathrm{SF}$ and retinal location or even the eye used during learning (Dosher \& Lu, 1999; Sowden et al, 2002), which suggested a possible locus of learning-induced plasticity in the early visual processing stages, such as the primary visual cortex (V1) or even earlier.

To uncover the neural substrate that mediates contrast detection learning dependent plasticity, creating an ideal animal model is the first step in trying to understand how contrast learning modifies the response properties of neurons in the visual pathway. To this end, we trained three cats to do the contrast detection learning using two-alternative forced choice method. Our main goal was to see whether contrast detection learning in cat showed a property similar to that in human subjects or not.

\section{Materials and Methods}

\subsection{Subjects}

Subjects were 3 adult male cats (age: $2-3$ years old; body weight: $2-2.5 \mathrm{~kg}$ ) with no apparent optical or retinal problems that would impair their visual functions. All cats were housed in one room and maintained on a 12 $\mathrm{h}: 12 \mathrm{~h} \mathrm{light/dark}$ cycle (lights on 7:00 am) with water available ad libitum. All of them could get as much food reward as they need through contrast detection practising for $2-3$ hours on each weekday and access food freely in the weekends. All animal treatments were strictly in accordance with the National Institutes of Health Guide for the Care and Use of Laboratory Animals.

\subsection{Psychological paradigms}

1.2.1 Apparatus The training apparatus was similar to that used by Vandenbussche (Vandenbussche \& Orban, 1983), De Weerd (De Weerd et al, 1990) and Orban (Orban et al, 1990) as previously described (Hua et al, 2005; Hua et al, 2007). Briefly, cats performed a required detection task displayed on a fixed displayer (CRT) and acquired food reward by pushing the correct nose key (right or left nose key). The task was to discriminate between two visual stimuli (see below), which were assigned a correspondence to right or left nose key. The distance from the CRT to the eyes was $57 \mathrm{~cm}$.

1.2.2 Stimuli and experimental procedure All visual signals were circular light spots with a diameter of $16 \mathrm{~cm}$ (equivalent to $8^{\circ}$ visual angle) and mean luminance of $19 \mathrm{~cd} / \mathrm{m}^{2}$. The program was written in MATLAB based on Psychtoolbox (Brainard, 1997). At first, cats were trained to discriminate binocularly between two randomly presented and temporally adjacent light spots, which contained sine-wave grating stimuli oriented either + or $-45^{\circ}$. The gratings SF was set at $0.1,0.2$ and $0.4 \mathrm{c} / \mathrm{deg}$ for cat 1 , cat 2 and cat 3 respectively (Fig 1A, B, $\mathrm{C})$. The mean luminance of grating stimuli was kept at $19 \mathrm{~cd} / \mathrm{m}^{2}$. All stimuli were presented in the center of the CRT. After $85 \%$ correct performance was attained for the first 6 consecutive days, we concluded that the cats had succeeded in discriminating between the two visual signals.

After conditioning training, monocular contrast detection learning (another eye was covered with a custom-made mask that blocked light) was performed subsequently. The trained SF was predetermined for each cat $(0.2 \mathrm{c} / \mathrm{deg}$ for cat $1,0.4 \mathrm{c} / \mathrm{deg}$ for cat 2 and $0.6 \mathrm{c} / \mathrm{deg}$ for cat3). We trained cats to do contrast detection using a 2-correct down/1-error up staircase method: The contrast of stimuli in each trial was either reduced to 0.9 times of that of previous trial if the cat made correct response in two consecutive trials or increased to 1.1 times if the cat 
made an incorrect response in the previous trial. This psychophysical algorithm tracks a threshold contrast required to reach a performance accuracy level of about $70.7 \%$.

In daily training session, each cat practised up to from 1000 to 1500 trials, which were generally arranged
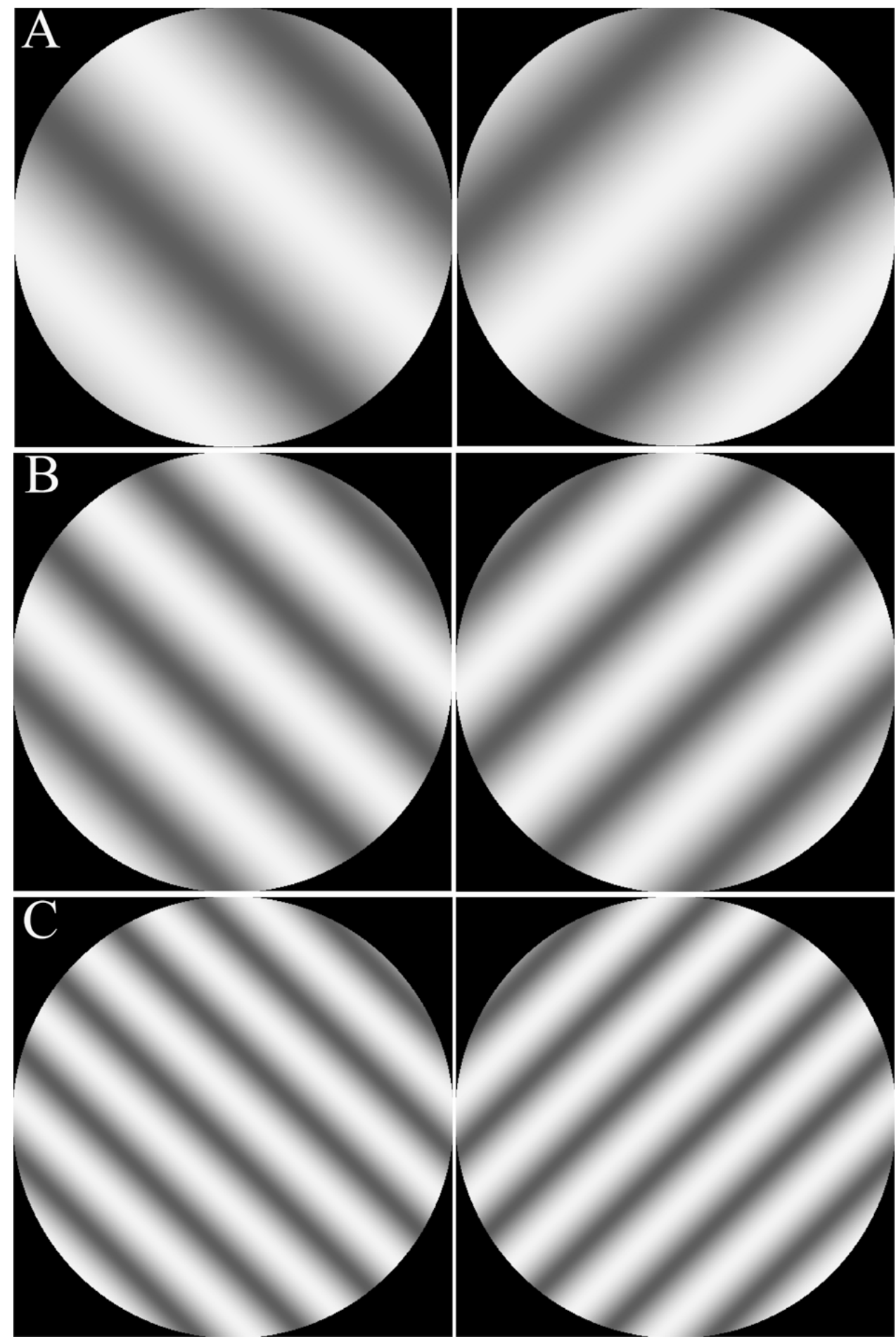

Fig. 1 Visual signal A, B and $\mathrm{C}$ are respectively used for conditioning training of cat1, cat2 and cat3

In either $\mathrm{A}$ or $\mathrm{B}$ or $\mathrm{C}$, stimuli are two light spots containing sine-wave gratings oriented + or $-45^{\circ}$. The gratings has a fixed mean luminance of $19 \mathrm{~cd} / \mathrm{m} 2$ and contrast of $80 \%$. The gratings SF was $0.1,0.2$ and $0.4 \mathrm{c} / \mathrm{deg}$ for cat 1 , cat 2 and cat 3 respectively.

in blocks of 100 trials per block (a few training blocks in cat 2 containing up to 400 trials). There was a 5 to 10 minutes rest between blocks. The experimenter triggered the first trial at the beginning of each block when everything was ready. Each trial started with a bright fixation dot $\left(0.1^{\circ}\right.$ visual angle $)$ appeared in the center
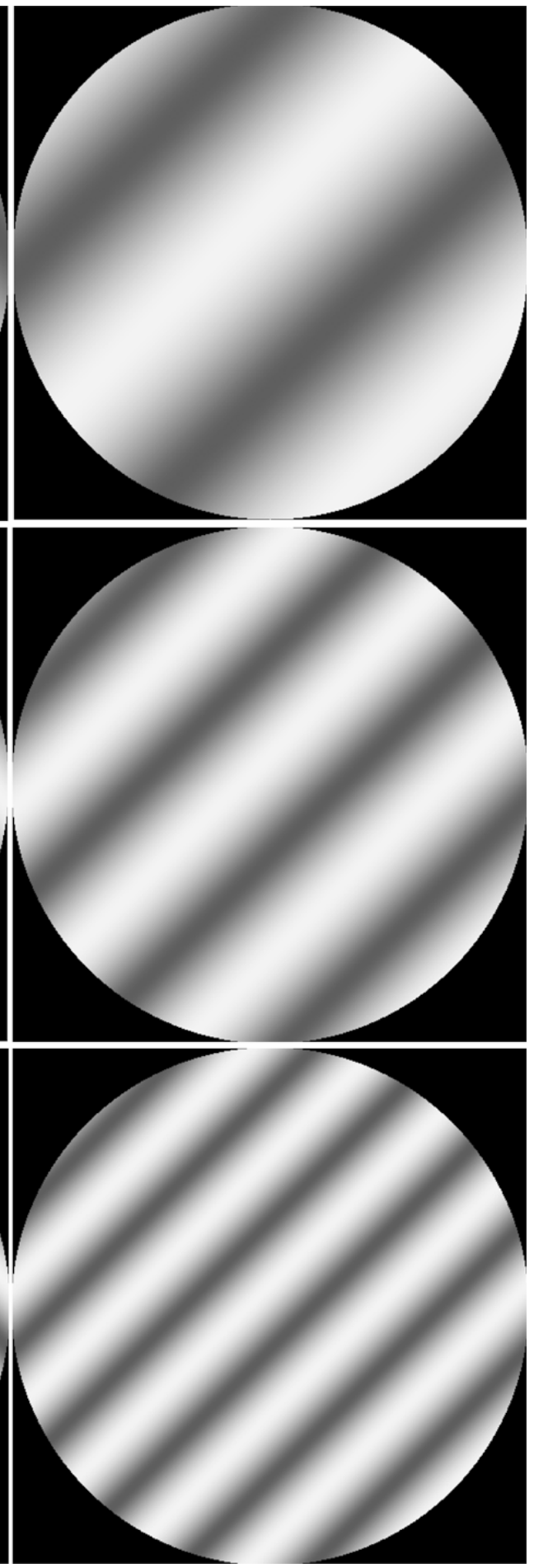$$
\text { . }
$$ 
of CRT for 1s. This was followed by a 4-second stimulus presentation with a 1-second response denied period (RDP) during which pushing the nose keys triggered no food reward. Inter-stimulus interval was set at 4 seconds between trials.

Contrast sensitivity functions (CSFs) for both trained and naïve eyes were monitored before and after contrast detection learning, with test SF covered 0.1, 0.2, $0.4,0.6,0.8,1.2$ and $1.6 \mathrm{c} / \mathrm{deg}(500-700$ trials for each $\mathrm{SF})$. The CSF measurement for trained and naïve eyes were conducted on alternate days. On each day, tests for different SF $(100-240$ trials for each SF) were arranged randomly. To familiarize naïve eyes with the learning task, 140 trials ( 7 blocks, 20 trials/block) of pre-test adaptation, using alternating SFs, were given on the first day of testing.

1.2.3 Data analysis The correct performance during conditioning training was measured in each training block, and the mean accuracy was obtained and monitored in daily training session. During contrast detection learning, the threshold contrast in each training block was tracked down using 2-correct down/1-error up staircase method and calculated automatically by a program. The mean threshold contrast value needed to discriminate between light spots containing either + or $-45^{\circ}$ grating stimulus was monitored by daily training session or calculated across blocks of a test. The contrast sensitivity at each SF was defined as log unit of the reciprocal of mean threshold contrast value. The CSFs before and after contrast detection learning were compared using one- or two-way ANOVA. A difference with $P<0.05$ was accepted as significant.

\section{Results}

\subsection{Perceptual learning of contrast detection}

All cats (cat1, cat2 and cat3) succeeded in discriminating between light spots containing grating stimuli oriented either + or $-45^{\circ}$ and attained $>85 \%$ correct performance after $3-4$ months of conditioning training.

Prior to contrast detection learning, CSFs were measured respectively for both eyes of each cat. Two-way ANOVA analysis indicated that the CSFs in each cat showed a main effect of SF [cat1: $F_{(6,134)}=$ $80.702, P<0.0001$; cat $2: F_{(6,128)}=87.884, P<$ 0.0001 ; cat $\left.3: F_{(6,133)}=130.186, P<0.0001\right]$, which is independent of eyes [Interaction of eye and SF: $F_{(6,134)}=$ $0.095, P=0.997$ in cat $1 ; F_{(6,128)}=0.678, P=0.668$ in cat 2 ; $F_{(6,133)}=1.631, P=0.143$ in cat3]. However, the contrast sensitivity at each SF (from 0.1 to $1.6 \mathrm{c} / \mathrm{deg}$ ) showed no significant difference between two eyes in each cat [cat1: $F_{(1,134)}=1.862, P=0.175$; cat $2: F_{(1,128)}=0, \quad P=0.998$; cat3: $\left.F_{(1,133)}=1.829, P=0.179\right]$. Therefore the CSFs from both eyes prior to contrast detection learning were combined for statistical analysis in each cat. As shown in the Gauss fitting curves, the CSF in each cat peaked around SF $0.4 \mathrm{c} / \mathrm{deg}$ and decreased gradually toward lower or higher SF (Fig. 2A, B, C), indicating that the optimal SF of visual stimuli that cat could detect was around $0.4 \mathrm{c} / \mathrm{deg}$.

Contrast detection learning lasts for $35-53 \mathrm{~d}$ till the stimulus threshold contrast needed to distinguish + $45^{\circ}$ gratings from $-45^{\circ}$ ones reached an asymptotic plateau. The SF chosen to train at is $0.2,0.4$ and 0.6 c/deg for cat1, cat 2 and cat 3 respectively. Fig 3 showed the contrast sensitivity promotion with learning in each cat. The learning effect was robust . Contrast sensitivity at the trained SF in the trained eye was improved by $196.1 \%\left[F_{(1,21)}=152.858, P<0.0001\right], 218.8 \%\left[F_{(1,42)}=\right.$ 474.607, $P<0.0001]$ and $172.2 \%\left[F_{(1,22)}=202.458\right.$, $P<0.0001$ ] in cat1, cat2 and cat3 respectively (Fig 3).

One or two days after contrast detection learning ( $53 \mathrm{~d}$ for cat $1,35 \mathrm{~d}$ for cat 2 and $44 \mathrm{~d}$ for dat 3 ), CSFs was measured again in three cats. As indicated by ANOVA analysis, significant increase in CSF was observed in the trained eye of each cat [cat1: $F_{(1,138)}=298.161, P<0.0001$; cat2: $F_{(1,144)}=253.209, P<0.0001$; cat $3: F_{(1,123)}=299.428$, $P<0.0001]$. The learning effect was highly dependent on stimulus SF [Main effect of SF: $F_{(6,138)}=199.407$, $P<0.0001$ in cat $1 ; F_{(6,144)}=290.231, P<0.0001$ in cat2; $F_{(6,123)}=231.754, P<0.0001$ in cat3; Interaction of learning and SF: $F_{(6,138)}=62.067, P<0.0001$ in cat1; $F_{(6,144)}=97.471, P<0.0001$ in cat2; $F_{(6,123)}=50.215$, $P<0.0001$ in cat3] (Fig. $2 \mathrm{~A}-\mathrm{C}$ ). Calculated from the Gauss fitting curves (fitting confidence $\geqslant 95 \%$ ), the learning effect, which was defined as the ratio of post-learning contrast sensitivity to pre-learning contrast sensitivity, in the trained eye was remarkably pronounced around the trained SF $(0.1-0.4 \mathrm{c} / \mathrm{deg}$ in cat $1,0.2-0.6 \mathrm{c} / \mathrm{deg}$ in cat 2 and $0.4-0.8 \mathrm{c} / \mathrm{deg}$ in cat 3 ) (Fig 2 D- F).

In addition, the learning effect in the trained eye could transfer to the untrained eye in each cat. After contrast detection learning, the contrast sensitivity at trained SF of naive eye improved by $88.7 \%\left[F_{(1,20)}=\right.$ 45.365, $P<0.0001], 89.9 \%\left[F_{(1,31)}=88.663, P<0.0001\right]$ and $96.4 \%\left[F_{(1,24)}=83.597, P<0.0001\right]$ in cat1, cat2 and cat3 respectively, an increase that was, however, 
significantly smaller than of the trained eye in each cat [cat1: $F_{(1,20)}=25.539, P<0.0001$; cat $2: F_{(1,40)}=180.08$; $P<0.0001$; cat $3: F_{(1,23)}=51.717, P<0.0001$ ] (Fig. $2 \mathrm{~A}-\mathrm{C}$ ). This inter-eye transfer effect could also be generalized to other SFs [cat1: $F_{(1,134)}=60.39, P<0.0001$; cat2: $F_{(1,125)}=$ 63.904, $P<0.0001$; cat3: $\left.F_{(1,139)}=92.18, P<0.0001\right]$ but was significant largely around the trained SF (from $0.1-0.4 \mathrm{c} / \mathrm{deg}$ in cat1, $0.2-0.6 \mathrm{c} / \mathrm{deg}$ in cat 2 and $0.4-.8$ c/deg in cat3) [cat1: $F_{(6,134)}=15.013, P<0.0001$; cat2: $F_{(6,125)}=23.709, P<0.0001$; cat $3: F_{(6,139)}=24.201, P<$
0.0001] (Fig. 2 D-F). Measured from the Gauss fitting curves, the learning effect of CSF in the naïve eye was also highest around the trained SF (from $0.1-0.4 \mathrm{c} / \mathrm{deg}$ in cat $1,0.2-0.6 \mathrm{c} / \mathrm{deg}$ in cat 2 and $0.4-0.8 \mathrm{c} / \mathrm{deg}$ in cat3)(Fig. 2 D - F).

Above results indicate that practising contrast detection can significantly increase contrast sensitivity of visual perception. The improvement, though characterizing a considerable specificity to the eye and the SF selected for training, exhibits partial generalizations to
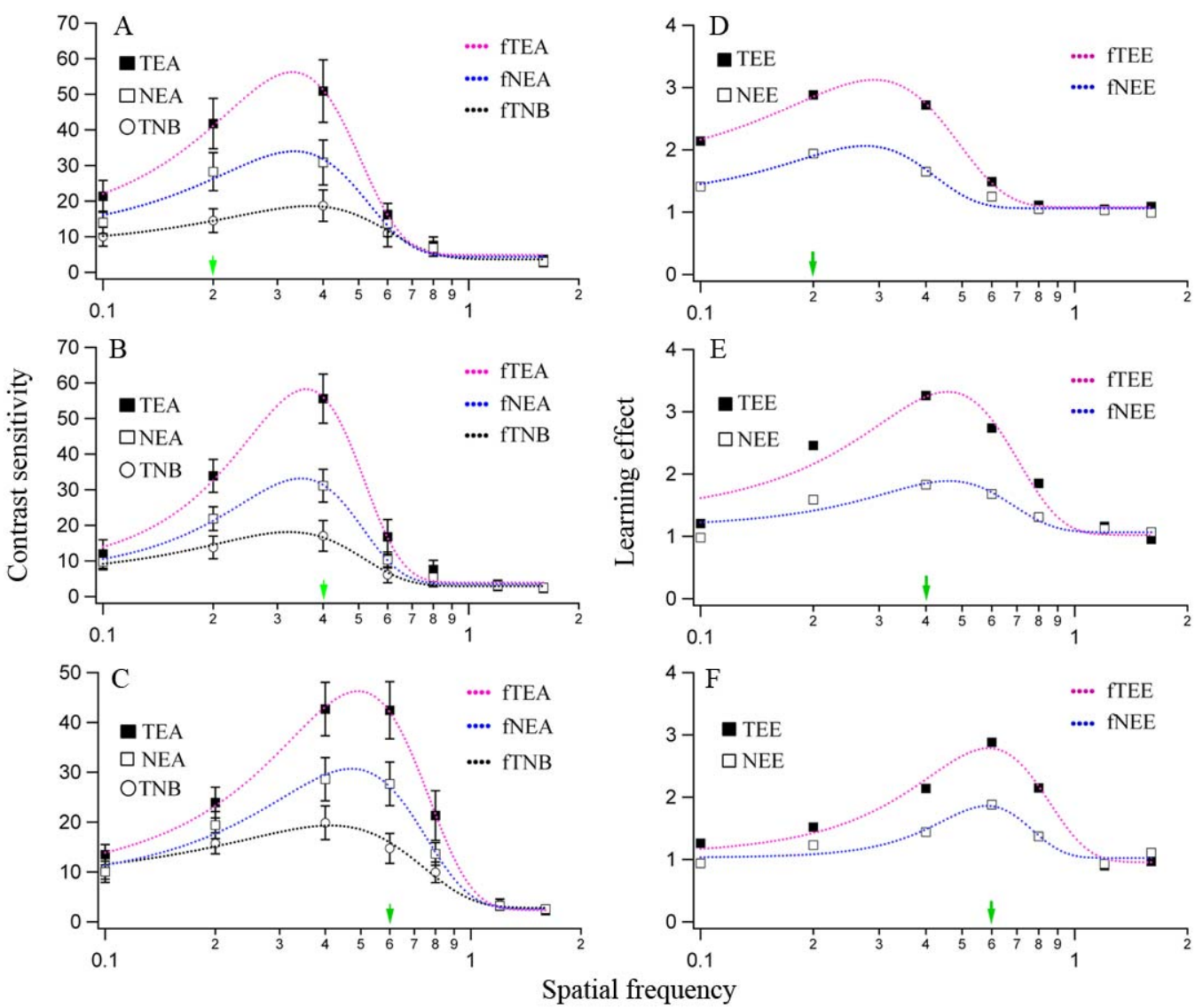

Fig. 2 Showing CSFs changes after more than one month (53 d in Cat1, $35 \mathrm{~d}$ in Cat2 and $44 \mathrm{~d}$ in Cat3) of contrast detection training as well as the learning effect in the trained and naïve eyes after learning relative to before learning

The CSFs measurement after contrast detection learning began from 55, 36 and $45 \mathrm{~d}$ after contrast detection training for Cat1, Cat2 and Cat3 respectively and lasted for $6-8 \mathrm{~d}$ in each cat. A/B/C show the CSFs measured for both eyes in cat1, cat2 and cat 3 respectively. Because there is no significant difference in CSFs of both eyes before contrast detection learning, the data from both eyes were combined. TNB (open circle) represents the combined CSFs of both eyes before learning. TEA (filled square) and NEA (open square) represent respectively the CSFs of the trained eye and naive eye after learning. FTEA (red dash), FNEA (blue dash) and FTNB (black dash) are Gauss fitting curve of TEA, NEA and TNB respectively. The CSFs of both eyes showed no significant difference in each cat before contrast detection learning. However, the CSFs of either trained or naive eye increased significantly after contrast detection learning, but the increase exhibited considerable dependency on the trained SF (cat1: $0.2 \mathrm{c} / \mathrm{deg}$; cat2: $0.4 \mathrm{c} / \mathrm{deg}$; cat3: $0.6 \mathrm{c} / \mathrm{deg}$ ) and was significantly higher in the trained eye than in the naive eye. D/E/F show the learning effect of both trained and untrained eyes in cat 1 , cat 2 and cat 3 respectively, and the learning effect is evident largely around the trained stimulus SF in each cat. TEE (filled square) and NEE (open square) represent the learning effect of the trained and naïve eye respectively. fTEE (red dash) and fNEE (blue dash) are Gauss fitting curve of TEE and NEE respectively. Green arrows indicate the stimulus SF used during contrast detection learning. 


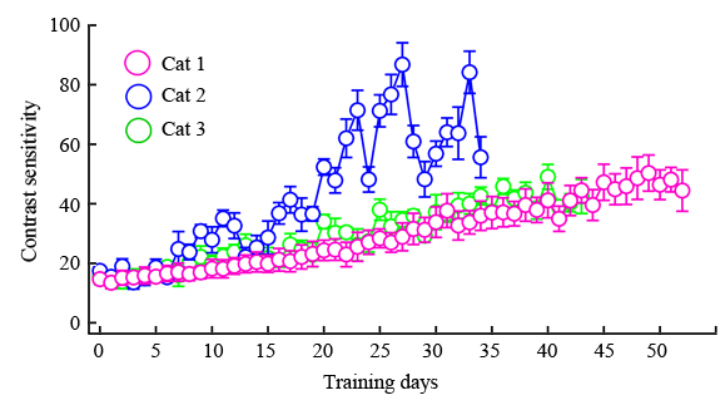

Fig. 3 Showing the daily contrast sensitivity changes with contrast detection learning in the trained eye of three cats (Cat1, Cat2 and Cat3)

The contrast sensitivity at the trained stimulus SF in the trained eye of each cat was significantly increased after more than one month of learning.

the naive eye and untrained SFs.

\subsection{Long-lasting learning effect}

Studies on visual perceptual learning in human and non-human primates has shown that the learning effect is long lasting. Whether contrast detection learning in cat exhibits a similar property has not been reported yet. To clarify this issue, we re-measured the CSFs in two trained cats (Cat 2 and Cat 3 ) about 5 months (162 d for Cat2, $151 \mathrm{~d}$ for Cat3) after contrast detection learning. Two-way ANOVA analysis showed that the re-measured $\mathrm{CSF}$ in the trained eye of both cats exhibited no significant difference from the CSF obtained 1 or $2 \mathrm{~d}$ after contrast detection learning [Cat2: $F_{(1,148)}=0.746$, $P=0.389$; Cat3: $\left.F_{(1,132)}=0.023, P=0.879\right]$. As indicated in Fig 5, the learning still displayed a highest gain around the trained SF $(0.2-0.6 \mathrm{c} / \mathrm{deg}$ in cat 2 and $0.4-0.8$ c/deg in cat3). Similarly, the re-measured CSF in the naïve eye of both cats were neither significantly different from the one monitered just after the contrast detection training period [Cat2: $F_{(1,134)}=0.117, P=0.733$; Cat3: $\left.F_{(1,126)}=0.97, P=0.327\right]$, and the learning effect was comparable to that previously observed (Fig 4A, B). This comparison strongly suggests that contrast detection learning can improve the visual contrast sensitivity in cat, and the learning effect is long-lasting.

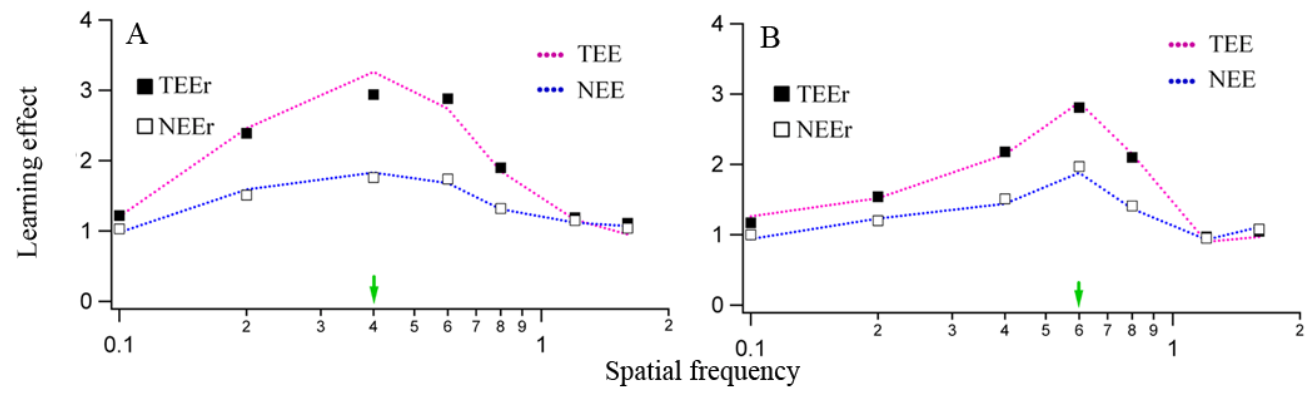

Fig. 4 Showing the learning effect measured in Cat2 (A) and Cat3 (B) about 5 months (162 d for Cat2, 151 $\mathrm{d}$ for Cat3) after the forced contrast detection training

TEE and NEE represent the learning gain in the trained and naïve eye respectively obtained after over a month of contrast detection learning. TEEr and NEEr show the learning gain value in the trained and naïve eye respectively re-measured about 5 months after contrast detection training. The green arrows indicate the stimulus SF used during training.

\section{Discussion}

Three cats (cat1, cat2 and cat3) succeeded in discriminating between light spots containing gratings oriented + or $-45^{\circ}$ and attained $>85 \%$ correct performance after $3-4$ months of conditioning training. Prior to contrast detection learning, CSFs of both eyes in each cat are comparable, and the CSF in each cat peaks around $0.4 \mathrm{c} / \mathrm{deg}$. Bisti and Maffei ever measured CSFs in two cats using stimuli with a large size (diameter $19^{\circ}$ visual acuity) but a low luminance $(2 \mathrm{~cd} / \mathrm{m} 2)$ level and found the peak CSF was around $0.2 \mathrm{c} / \mathrm{deg}$, which was in good agreement with the CSF evaluation using visually evoked potentials measurement (Berkley \& Watkins,
1973; Bisti \& Maffei, 1974; Campbell et al, 1973). In this study, a smaller (diameter $8^{\circ}$ visual acuity) but higher luminance $\left(19 \mathrm{~cd} / \mathrm{m}^{2}\right)$ stimuli were used, and the peak CSF we observed in each of the three cats was around $0.4 \mathrm{c} / \mathrm{deg}$, which was higher than that reported by Bisti and Maffei but could be reconciled by previous findings that the peak CSF increased with stimulus mean luminance and decreased with stimulus size (Jarvis \& Wathes, 2008). The CSFs amplitude we obtained from each cat were much lower than that measured by Pasternak and Merigan who used stimuli with a luminance $\left(16 \mathrm{~cd} / \mathrm{m}^{2}\right)$ very close to ours but with a size $\left(11-14^{\circ}\right.$ visual acuity) slightly larger than ours (Pasternak \& Merigan, 1981). This large difference may 
comes from the following factors: 1) The task in our experiment is to discriminate between + and $-45^{\circ}$ oriented gratings near threshold contrast, which is much difficult than distinguishing vertical sinusoidal gratings from uniform fields of the same mean luminance as described by Pasternak and Merigan; 2) In this study, the CSFs were constructed based on an average of $7-14$ blocks $(50-80$ trials per block) of contrast threshold measurement per each SF. On each testing day, measurement at different SFs were pseudorandomly interleaved. However, Pasternak and Merigan obtained the CSF by continuing measuring till asymptotic performance was reached at each SF, which would likely over-estimate the contrast sensitivity at each SF. For example, we did ever get much higher contrast sensitivity value in cat 2 during a few of training days when several hundreds of continuous trials were applied (Fig. 3); 3) Pasternak and Merigan obtained the CSF based on a summation from both eyes of the cat. However, we just measured the monocular CSF, which was predicted to be 1.414 times lower than the binocular value even under the same test stimulus conditions (Legge, 1984a, b).

Although there are variations in the measuring result concerning the peak CSF and CSF amplitude, the CSF of cat is indeed different from that of human and other species (Jarvis \& Wathes, 2008). For example, under the same stimulus luminance level, the peak CSF of cats was much less, and their CSF was located at lower SF domain than that of humans (Blake et al, 1974; Pasternak \& Merigan, 1981). Factors causing species-specific CSF, including peak CSF and SF domain, could be attributed partially to different retinal cell distribution patterns but needs to be explored extensively in subsequent studies (Jarvis \& Wathes, 2008).

Following contrast detection training of more than one month, the contrast sensitivity of trained eye at trained SF increased by $196.1 \%, 218.8 \%$ and $172.2 \%$ in cat 1 , cat 2 and cat 3 respectively. The improvement was robust as opposed to similar learning in human fovea (Polat et al, 2004; Zhou et al, 2006) or parafovea (Sowden et al, 2002). The learning effect showed an evident dependency on the stimulus SF used for learning but could partially generalize to SFs around the trained SF. This indicates that contrast detection is likely mediated through SF channels, which depends directly on the receptive field dimensions of visual cortical cells. Based on this property of contrast detection, attemps to rehabilitate the visual contrast sensitivity in patients, such as patients with amblyopia (Levi, 2005) and primary visual cortex damage, is better to apply a training procedure containing visual stimuli with a broadband SFs.

The learning could transfer to the untrained fellow eye. After learning, CSF at trained SF in naive eye was improved by $88.7 \%, 89.9 \%$ and $96.4 \%$ relative to pre-learning in cat1, cat2 and cat3 respectively, a somewhat minor increase compared with what we observed in the trained eye. Sowden et.al. have reported that perceptual learning of contrast detection in human parafovea was specific for stimulus SF and retinal location but not stimulus orientation (Sowden et al, 2002). In addition, they found that the enhancement in contrast sensitivity in the trained eye could transfer to untrained eye although the average learning effect in the trained eye was $45 \%$ greater than that in the contralateral eye. Our results achieved in cat basically resemble that observed by Sowden et.al in human subjects. The CSF promotion resulted from learning showed some specificity to the trained eye but could partially generalize to the naïve eye, which suggested a main learning plasticity at early stages of visual information processing, such as the primary visual cortex and lateral geniculate nucleus, accompanied by a minor plasticity that might occur after information from both eyes merged together. Subsequent studies that aim to uncover the neural correlates underlying learning-induced contrast sensitivity improvement need to pay attentions to nucleus at both early and late stages of visual information processing.

In summary, contrast detection learning can remarkably improve contrast sensitivity of cat to visual stimuli. The learning effect, which is proven to be long-lasting, shows an evident specificity to the eye and SF used for learning but can partially transfer to the naïve eye and SFs around the trained SF. These properties of contrast detection learning tally well with reports in human subjects (Sowden et al, 2002), which indicates that the cat species is qualified to be used as an animal model in probing the neural correlates that underlies learning-induced contrast sensitivity promotion and other visual perceptual learning.

Acknowledgements: The authors are greatly indebted to Professor Yi-Feng Zhou in University of Science and Technology of China and Professor Randolph Blake for the generous donation of the cat behavioural apparatus, without which the study would have been impossible. 


\section{References:}

Altangerel U, Spaeth GL, Steinmann WC. 2006. Assessment of function related to vision (AFREV) [J]. Ophthalmic Epidemiol, 13(1): 67-80.

Berkley MA, Watkins DW. 1973. Grating resolution and refraction in the cat estimated from evoked cerebral potentials [J]. Vision Res, 13(2): 403-415.

Bisti S, Maffei L. 1974. Behavioural contrast sensitivity of the cat in various visual meridians [J]. J Physiol, 241(1): 201-210.

Blake R, Cool SJ, Crawford ML. 1974. Visual resolution in the cat [J]. Vision Res, 14(11): 1211-1217.

Brainard DH. 1997. The psychophysics toolbox [J]. Spat Vis, 10(4): 433-436.

Campbell FW, Maffei L, Piccolino M. 1973. The contrast sensitivity of the cat [J]. J Physiol, 229(3): 719-731.

De Weerd P, Vandenbussche E, Orban GA. 1990. Bar orientation discrimination in the cat [J]. Vis Neurosci, 4(3): 257-268.

Dosher BA, Lu ZL. 1998. Perceptual learning reflects external noise filtering and internal noise reduction through channel reweighting [J]. Proc Natl Acad Sci USA, 95(23): 13988-13993.

Dosher BA, Lu ZL. 1999. Mechanisms of perceptual learning [J]. Vision Res, 39(19): 3197-3221.

Ghose GM, Yang T, Maunsell JH. 2002. Physiological correlates of perceptual learning in monkey V1 and V2 [J]. J Neurophysiol, 87(4): 1867-1888.

Gilbert CD. 1994. Early perceptual learning [J]. Proc Natl Acad Sci USA, 91(4): 1195-1197.

Ginsburg AP. 2006. Contrast sensitivity: determining the visual quality and function of cataract, intraocular lenses and refractive surgery [J]. Curr Opin Ophthalmol, 17(1): 19-26.

Gray R, Regan D. 1998. Spatial frequency discrimination and detection characteristics for gratings defined by orientation texture [J]. Vision Res, 38(17): 2601-2617.

Grove PM, Regan D. 2002. Spatial frequency discrimination in cyclopean vision [J]. Vision Res, 42(15): 1837-1846.

Hua TM, Mei B, Wang HT, Liu ZQ, Sun QY. 2005. Oriented grating detection learning does not change the orientation selectivity of dLGN neurons in cat [J]. Acta Zool Sin, 51(2): 274-279.

Hua TM, Wan A, Wang SY, Mei B, Sun QY. 2007. Perceptual learning of grate orientation discrimination in cats [J]. Zool Res, 28(1):9 5-100. (in Chinese)

Jarvis JR, Wathes CM. 2008. A mechanistic inter-species comparison of spatial contrast sensitivity [J]. Vision Res, 48(21): 2284-2292.

Karni A, Sagi D. 1991. Where practice makes perfect in texture discrimination: Evidence for primary visual cortex plasticity [J]. Proc Natl Acad Sci USA, 88(11): 4966-4970.

Lages M, Treisman M. 1998. Spatial frequency discrimination: Visual long-term memory or criterion setting [J]. Vision Res, 38(4): 557-572.

Legge A. 1984a. Binocular contrast summation-I. Detection and discrimination [J]. Vision Res, 24: 373-383.

Legge A. 1984b. Binocular contrast summation - II. Quadratic summation [J]. Vision Res, 24: 385-395.

Levi DM. 2005. Perceptual learning in adults with amblyopia: A reevaluation of critical periods in human vision[J]. Dev Psychobiol, 46(3): 222-232.

Matthews N, Liu Z, Geesaman BJ, Qian N. 1999. Perceptual learning on orientation and direction discrimination [J]. Vision Res, 39(22): 3692-3701.

Matthews N, Liu Z, Qian N. 2001. The effect of orientation learning on contrast sensitivity [J]. Vision Res, 41(4): 463-471.

Niebauer CL, Christman SD. 1999. Visual field differences in spatial frequency discrimination [J]. Brain Cogn, 41(3): 381-389.

Orban GA, Vandenbussche E, Sprague JM, De Weerd P. 1990. Orientation discrimination in the cat: a distributed function [J]. Proc Natl Acad Sci USA, 87(3): 1134-1138.

Pasternak T, Merigan WH. 1981. The luminance dependence of spatial vision in the cat [J]. Vision Res, 21(9): 1333-1339.

Piermarocchi S, Sartore M, Bandello F, Lanzetta P, Brancato R, Garattini L, Lumbroso B, Rispoli M, Pece A, Isola V, Pulazzini A, Menchini U, Virgili G, Tedeschi M, Varano M. 2006. Quality of vision: A consensus building initiative for a new ophthalmologic concept [J]. Eur J Ophthalmol, 16(6): 851-860.

Polat U, Ma-Naim T, Belkin M, Sagi D. 2004. Improving vision in adult amblyopia by perceptual learning [J]. Proc Natl Acad Sci USA, 101(17): 6692-6697.

Raiguel S, Vogels R, Mysore SG, Orban GA. 2006. Learning to see the difference specifically alters the most informative V4 neurons $[\mathrm{J}]$. J Neurosci, 26(24): 6589-6602.

Riusala A, Sarna S, Immonen I. 2003. Visual function index (VF-14) in exudative age-related macular degeneration of long duration $[\mathrm{J}]$. Am J Ophthalmol, 135(2): 206-212.

Rivest J, Boutet I, Intriligator J. 1997. Perceptual learning of orientation discrimination by more than one attribute $[\mathrm{J}]$. Vision Res, 37(3): 273-281.

Sally SL, Gurnsey R. 2003. Orientation discrimination in foveal and extra-foveal vision: effects of stimulus bandwidth and contrast $[\mathrm{J}]$. Vision Res, 43(12): 1375-1385.

Schoups A, Vogels R, Qian N, Orban G. 2001. Practising orientation identification improves orientation coding in V1 neurons [J]. Nature, 412(6846): 549-553.

Sowden PT, Rose D, Davies IR. 2002. Perceptual learning of luminance contrast detection: specific for spatial frequency and retinal location but not orientation [J]. Vision Res, 42(10): 1249-1258.

Vandenbussche E, Orban GA. 1983. Meridional variations in the line orientation discrimination of the cat [J]. Behav Brain Res, 9(2): 237-255.

Vimal RL. 2002. Spatial frequency discrimination: a comparison of achromatic and chromatic conditions [J]. Vision Res, 42(5): 599-611.

Yang T, Maunsell JH. 2004. The effect of perceptual learning on neuronal responses in monkey visual area V4 [J]. $J$ Neurosci, 24(7): 1617-1626.

Zhou Y, Huang C, Xu P, Tao L, Qiu Z, Li X, Lu ZL. 2006. Perceptual learning improves contrast sensitivity and visual acuity in adults with anisometropic amblyopia [J]. Vision Res, 46(5): 739-750. 